REVISTA CIENCIAS BIOMÉDICAS

LAS LETRAS DEL MAESTRO

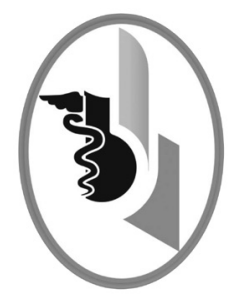

\title{
CONTRIBUCIÓN AL TRATAMIENTO DE LA FILARIASIS POR EL SALVARSÁN*
}

\author{
SALVARSÁN CONTRIBUTION TO THE FILARIASIS \\ TREATMENT
}

Gómez-Sánchez Julio F. ${ }^{1}$

Obregón-Flórez Manuel F. ${ }^{1}$

\section{RESUMEN}

Las letras del maestro es la sección de la Revista Ciencias Biomédicas que recoge escritura científica del ayer, realizada por profesionales médicos de la Universidad de Cartagena en Colombia. En este número se reproduce un documento de 98 años de antigüedad donde quedó plasmada la capacidad de análisis clínico de los eventos mórbidos. El texto posee estructuración de corte analítico que deja ver a profesionales con conocimientos profundos sobre una patología que era endémica de Cartagena en esos momentos, debido al consumo de agua que era recogida y conservada en aljibes y albercas. También se citan autores de elevado reconocimiento científico, lo que demuestra la cercanía de los autores a publicaciones de importancia mundial. El nivel de actualización es llamativo y la capacidad para presentar tesis y argumentos basados en la observación médica son elementos constantes. Por medio de los casos clínicos de la experiencia de los autores, se desglosa una patología (filariasis) y una propuesta terapéutica (Salvarsán $($ ). Rev.cienc.biomed. 2016;7(2):376-390.

\section{PALABRAS CLAVE}

Historia de la medicina; Enfermedades trasmisibles; Tratamiento farmacológico.

\section{SUMMARY}

The words of the master is a section in the Revista Ciencias Biomédicas that recognizes scientific articles made by medical professionals at Universidad de Cartagena -

* Reproducido de Revista Médica de Bogotá. Órgano de la Academia Nacional de Medicina. Serie XXXVI. Bogotá. Imprenta Nacional. 1918. Trabajo presentado en el Tercer Congreso Nacional de Medicina, reunido en Cartagena. Colombia.

1 Médico. Profesor. Universidad de Cartagena. Colombia. 
Colombia in the past. This issue reproduces a 98-year-old document where the capacity for clinical analysis of the morbid events was captured. This document has an analytic structure that show the deep knowledge about an endemic pathology in Cartagena during that period due to the consumption of water that was collected and conserved in cisterns and pools. Also authors of high scientific recognition are cited, which shows the proximity of the authors to publications of world-wide importance. The level of updating is striking and the ability to present theses and arguments based on medical observation are constant elements. By means of the clinical cases of the experience of the authors, a pathology (filariasis) and a therapeutic proposal are described (Salvarsán $®$ ). Rev. cienc.biomed. 2016;7(2):376-390.

\section{KEYWORDS}

History of medicine; Communicable diseases; Drug therapy.

\section{NOTA DEL EDITOR}

La Revista Ciencias Biomédicas para conmemorar los 105 años del inicio de la popularización del Salvarsán como estrategia terapéutica reproduce el texto de los médicos que ejercieron en Cartagena, Julio F. Gómez Sánchez y Manuel Francisco Obregón Flórez, en el cual realizan la presentación de más de un decena de casos clínicos con diferentes expresiones de la filariasis y las respuestas al tratamiento con el uso de arsenicales por diferentes vías y concentraciones. El documento a punto de ser un texto médico centenario fue publicado por la Revista Médica de Bogotá, órgano de la hoy Academia Colombiana de Medicina, en el año 1918 y además, fue presentado de forma oral dentro del Tercer Congreso Médico Nacional de Colombia celebrado en Cartagena en ese mismo año. Fue el primer evento científico realizado formalmente en esta ciudad, de reconocido impacto y trascendencia en la historia de la medicina colombiana y que está próximo a cumplir los primeros cien años de su realización.

En los inicios del siglo XX Cartagena presentaba una elevada prevalencia de filariasis, que como se observa en los casos presentados, se caracterizaba por diferentes expresiones clínicas, una de las más frecuentes era la elefantiasis. Hasta bien avanzada la séptima década de ese siglo todavía se veían en las calles personas afectadas por la elefantiasis y eran frecuentes los casos de compromiso inguinoescrotal, en cuyo caso los afectados eran denominados potrosos.
La filariasis linfática o elefantiasis es una enfermedad tropical infecciosa, producida por la transmisión de parásitos denominados filarias a través de mosquitos. La infección se adquiere usualmente en la infancia y causa daños en el sistema linfático. Los cuadros dolorosos y las desfiguraciones como consecuencia del linfedema e inflamación aparecen tardíamente y pueden causar discapacidad permanente, con impacto adverso psicológico y social, con la consiguiente estigmatización.

La elefantiasis es causada por nematodos de la familia Filarioidea, donde la Wuchereria bancrofti es responsable del $90 \%$ de los eventos; los otros son: La Brugia malayi y La Brugia timori. Los gusanos crecen entre seis y ocho años y son capaces de producir millones de pequeñas larvas, también denominadas microfilarias, que circulan en el torrente sanguíneo. Los mosquitos se infectan con microfilarias al picar a un portador. Ellas maduran en el mosquito y se convierten en larvas infecciosas. Cuando el mosquito infectado pica a otras personas, las larvas son depositadas en la piel, desde donde penetran el organismo, pasan a los vasos linfáticos, se desarrollan y se vuelven gusanos adultos que producen microfilarias, mientras dañan las estructuras linfáticas. Diferentes tipos de mosquitos están involucrados en ese ciclo como Culex, Anopheles y Aedes.

El Salvarsán $®$, derivado arsenical, fue llamado, incluso en elevados niveles científicos, "la bala mágica", una sustancia capaz de actuar de forma específica sobre microorganismos 
REPUBLICA DE COLOMBIA

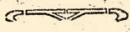

\section{Revista Médica}

\section{de Bogotá}

Organo de la Academia Nacional de Medicina.

SERIE XXXVII

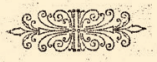

Bocotá

Imprenta Nacional

1918 sin afectar las estructuras corporales. Posteriormente sería el punto de partida para el desarrollo de la farmacología. El 10 de abril de 1910, Paul Ehrlich presentó su "compuesto 606" en el XXVII Congreso Alemán de Medicina Interna, en Weisbaden. El "compuesto 606" fue denominado Salvarsán ${ }^{\circledR}$, aunque era conocido popularmente como "606" o "Ehrlich-Hata 606", en reconocimiento a sus creadores. También fue denominado Arsphenamine. Un par de años después, Ehrlich presentó el 914 o Neosalvarsán, más soluble, fácil de usar y no perdía eficacia. Ehrlich logró eliminar gérmenes causantes de enfermedad sin lesionar al organismo mediante la inyección de un producto en la sangre. Su conjunto de trabajos significó su gloria y el comienzo de una etapa fundamental para la farmacología y la terapéutica.

La historia del desarrollo del Salvarsán $\mathbb{R}$ es importante en la historia de la medicina universal, puesto que permitió aprender

\section{CONTRIBUCION}

al tratamiento de la filariosis por el salvarsán, por los doctores JULIO F. GOMEZ SANCHEZ y MANUEL F. OBREGON, de Cartagena.

(Trabajo presentado a1 tercer Congreso Nacional de Medicina, reunido en Cartagena).

Para todo práctico que ejerza en Cartagena, la nosologia de esta importantísima ciudad reviste caracteres de tan grande importancia, que preocuparse por la solución de los grande importancia, que preocuparse por la solución de los
problemas patológicos que aquí se presentan es casi llenar un deber profesional.

Eutre las cuestiones que aquí se ofrecen al estudio del observador aparece en primer término la filariosis, entidad patológica que existe en estado endémico en la ciudad, y que estudiada suficientemente en su etiología, su bistoria, su nosogenia, su distribución geográfica, etc.... todavía es un enigma en lo que a tratamiento se refiere.

Ahora bien: el conocimiento que teníamos del atoxil

como cuerpo arsenical orgánico, que sin riesgo de intoxicación ejerce favorable influencia sobre ciertas espirilosis; sus aplicaciones, dondequiera que el arsénico se halla indicado, y las analogías químicas y terapéuticas de aquel compuesto con el salvarscin, nos hicieron suponer casi simultáneamente $y \sin$ que el uno tuviera conocimiento de las inrectigaciones del otro, que este último medicamento bien eariga poda utilizarse ya que do sobre los protozoes, espirilas, hematozoarios, etc., se hiciera sentir igualmente sobre los nematodas de Brancoft $y$ sus múltiples manifestaciones morbosas.

Bajo el influjo de estas ideas empezó cada uno de nostros a bacer separadamente ensayos de tratamiento de la flariosis por el arsenobenzol, y habiendo coincidido en reviltados y conocido reciprocamente a ́́ltima hora la intenón que cada uno tenia de informar al tercer cor ia de simplificación y por obra de cordialidad profesional, esumir nuestras observaciones en un solo trabajo, que es que tenemos la honra de presentar hoy.

- De todos es sabido que la filariosis está constituída por

se conjunto de perturbaciones anatomoclínicas provoca-

que los medicamentos se mueven entre el beneficio y el riesgo, entre la curación y el agravamiento. Facilitó la aceptación de conceptos que hoy día son esenciales y obvios en farmacología, mientras que fue sendero para el desarrollo de derivados y caldo para el nacimiento de lo que Paul Ehrlich Ilamó la quimioterapia, que ya cuenta dentro de la historia de la humanidad

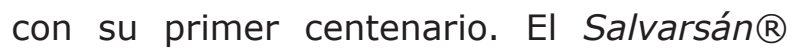
se dejó de utilizar junto con los demás integrantes de los arsenicales cuando en la mitad del siglo XX llegó la era antibiótica, pero abrió oportunidad para otras "balas mágicas", las sulfamidas, otros antibióticos numerosos productos orgánicos con eficacia terapéutica. La película Dr. Ehrlich's Magic bullet, se estrenó el 2 de marzo de 1940 y recuerda la vida del investigador, su proceso investigativo, las barreras y obstáculos que debió vencer. Este importante y agudo investigador fue galardonado en 1908 con el Premio Nobel. 
En la actualidad, el esquema propuesto por la Organización Mundial de la Salud para tratar la elefantiasis consiste en administrar a la población en riesgo, a manera de quimioprofilaxis masiva, una dosis anual combinada de 400/mgs de albendazol 400/ mg más $150-200 \mathrm{mcg} / \mathrm{Kg}$ de ivermectina o 6 $\mathrm{mgs} / \mathrm{Kd}$ de dietilcarbamazina.

En Cartagena hace muchos años que no se identifican al parecer casos de elefantiasis, por lo que ha dejado de ser endémica, sin embargo se considera que más de 36 millones de personas en el mundo presentan manifestaciones crónicas de la enfermedad. Casi 30 millones de hombres padecen enfermedad genital o potra, y cerca de 20 millones de personas experimentan linfedema. La Organización Mundial de la

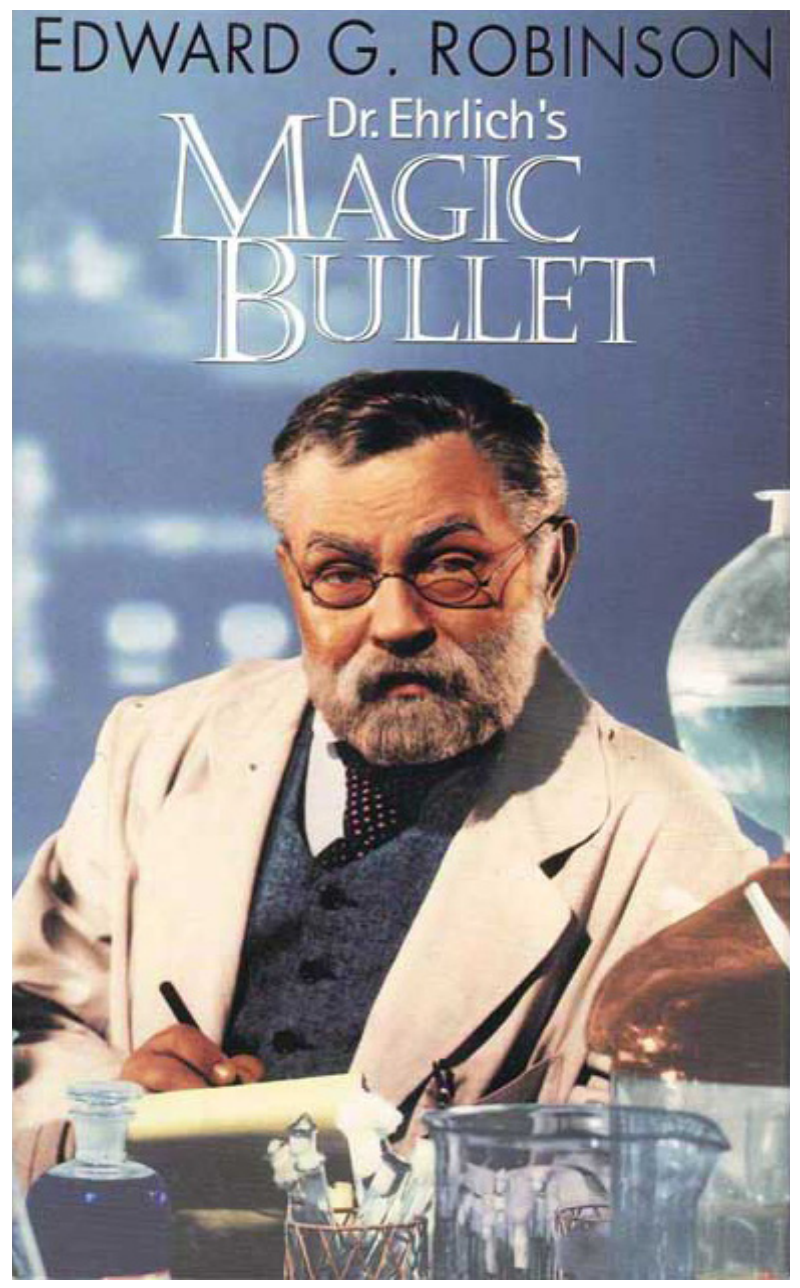

Afiche promocional de la Pelicula Dr. Ehrlich's Magic bullet. http://www. historiadelamedicina.org/606Expo/ehrlich2.htmI
Salud, en el año 2000, dio inicio al Programa Mundial para Eliminar la Filariasis Linfática, en una valoración del año 2012 reafirmó el plazo del año 2020 para lograr la eliminación de la entidad a nivel mundial. A pesar de que se acerca esa meta, la quimioprofilaxis se requería en más de cincuenta países para finales del año 2016, con la existencia de zonas endémicas donde aún no se ha iniciado.

Para el 2020 se habrá conseguido el éxito si los pacientes tienen el tratamiento para los episodios de linfangitis, oportunidad temprana de diagnóstico y tratamiento, cirugía para corregir las deformidades, tratamiento para las personas infectadas, quimoprevención, mejoría sanitaria, en las condiciones de vida y un plan amplio para la destrucción de los vectores.

Revistas prestigiosas como New England Journal of Medicine y Lancet, en el año 1911, publicaron las primeras apreciaciones y recomendaciones sobre el Salvarsán $®$. Las afirmaciones presentes en el texto de Gómez y Obregón permiten identificar el nivel de actualización y cómo con prontitud se podía recibir la actualización de las acciones médicas de esa época. La capacidad de análisis, el juicio clínico, el racionamiento lógico y la disponibilidad para abordar el enfoque médico quedan claros a lo largo del texto, que pese a haber sido escrito hace cien años, permite ilustrar y ser ejemplo de cómo realizar escritura científica.

Los autores con prosa científica exponen y argumentan sus observaciones clínicas, luego de realizar la administración del novísimo medicamento a sus pacientes. Presentan una lista de sus limitaciones, se hacen preguntas, reflexionan en cada párrafo y con iniciativa de ciencia acorde a esa época, recomiendan que el medicamento tendría una importante indicación para el manejo de las filariasis, tal vez no señalada anteriormente. Revista Ciencias Biomédicas invita a la lectura y a observar el pensamiento científico de un par de médicos que atendieron pacientes de Cartagena, Colombia cuando se cursaban las primeras décadas del siglo XX, y la filariasis 


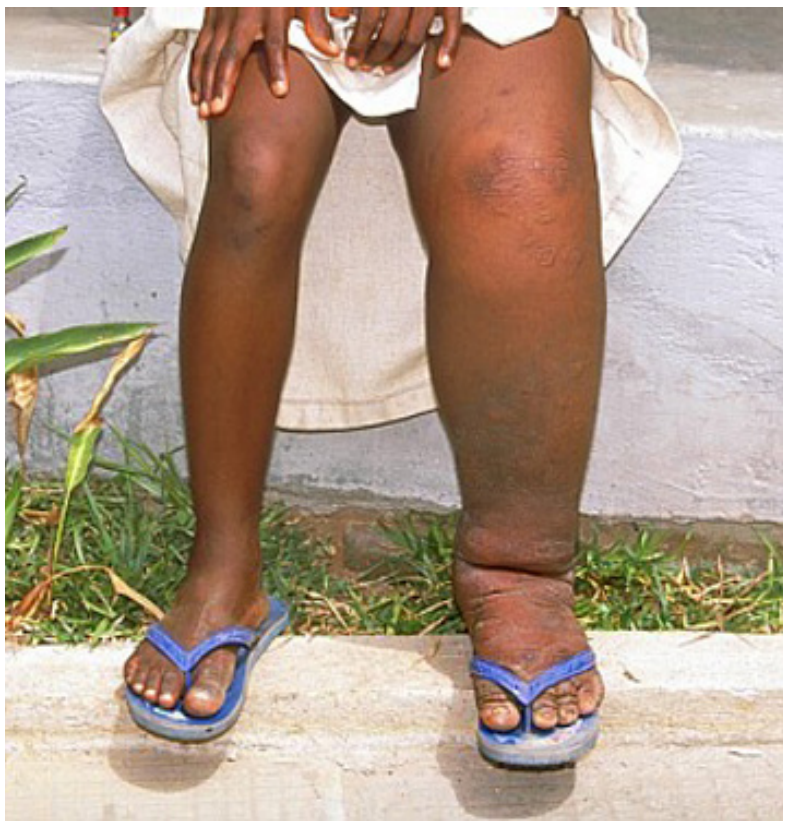

http://diseasespictures.com/wp-content/ uploads/2012/12/Elephantitis-3.jpg

causaba cuadros severos de elefantiasis; y las potras eran importante motivo para discriminación social.

\section{CONTRIBUCCIÓN AL TRATAMIENTO DE LA FILARIASIS POR EL SALVARSÁN}

Para todo práctico que ejerza en Cartagena, la nosología de esta importantísima ciudad reviste caracteres de tan grande importancia, que preocuparse por la solución de los problemas patológicos que aquí se presentan es casi llenar un deber profesional.

Entre las cuestiones que aquí se ofrecen al estudio del observador aparece en primer término la filariosis, entidad patológica que existe en estado endémico en la ciudad, y que estudiada suficientemente en su etiología, su historia, su nosogenia, su distribución geográfica, etc., todavía es un enigma en lo que a tratamiento se refiere.

Ahora bien: el conocimiento que teníamos del atoxil como cuerpo arsenical orgánico, que sin riesgo de intoxicación ejerce favorable influencia sobre ciertas espirilosis; sus aplicaciones, donde quiera que el Arsénico se haya indicado, y las analogías químicas y terapéuticas de aquel compuesto con el salvarsán, nos hicieron suponer casi simultáneamente y sin que el uno tuviera conocimiento de las investigaciones del otro, que este último medicamento bien podría utilizarse en el tratamiento de la filariosis, ya que era lógico suponer que la acción electiva de este preparado sobre los protozoes, espirilas, hematozoarios, etc., se hiciera sentir Igualmente sobre los nematodas de Brancoft y sus múltiples manifestaciones morbosas.

Bajo el influjo de estas ideas empezó cada uno de nosotros hacer separadamente ensayos de tratamientos de la filariosis por el arsenobenzol, y habiendo coincidido en resultados y conocido recíprocamente a la última hora la intención de cada uno tenía de informar al Tercer congreso médico Nacional de dicho resultados, hemos resuelto, por vía de simplificación y por obra de cordialidad profesional, resumir nuestras observaciones en un solo trabajo, que es el que tenemos la honra de presentar hoy.

De todos es sabido que la filariosis está construida por ese conjunto de perturbaciones anatomoclínicas provocadas por el parasito nematodas, llamado filaria, del cual se conocen a los menos seis variedades; más sin tener en cuenta la nosografía de tal entidad morbosa, y más que todo por falta de medios adecuados para trabajos de tal naturaleza, nuestras investigaciones se han dirigido especialmente a la filaria nocturna, que parece sea la especie más frecuente en Cartagena.

Encontrando aquí la mayor parte de las manifestaciones patológicos de la filariosis, ocasiones muy propicias hemos tenido para someter a prueba nuestro tratamiento, y los ensayos hechos en casi todas esas manifestaciones aparecen con su resultado, en las siguientes observaciones:

\section{LINFOESCROTO}

Caso I (Observación del doctor Gómez Sánchez). N.N., de treinta y dos años de edad, soltero, natural de Cartagena, de raza blanca y construcción fuerte. Antecedente hereditario, su madre murió de filariosis. Antecedentes patológicos. Desde hace algunos años sufre de <erisipela genital> cuyos accesos, que se hacían cada vez 
más frecuente (uno o dos por mes), lo imposibilitaban para toda clase de trabajo. Hechos el diagnóstico de la filariosis, y conociendo las propiedades fisiológicas del compuesto Arsenical en que nos ocupamos, practicamos una inyección intravenosa de 0.60 centigramos de salvarsán (606). La reacción general fue bastante violenta. Efecto de la inyección: no volvieron a repetirse los accesos febriles, que era incesantes y rebeldes de todo tratamiento, disminuyó no sólo la irritabilidad de la piel afectada sino el estado de hiperestesia, tan molesto que muchos enfermos; el estado mejoró notablemente. El enfermo no ha tenido hasta presidente ninguna recidiva.

Caso II (Observaciones del doctor Gómez Sánchez). N.N., de veinte años de edad natural de Cartagena, de constitución débil y anemia considerable. Antecedente hereditario sin importancia. Antecedentes personales. Desde la edad de la pubertad comenzó a notar un engrosamiento doloroso inguinoescrotal especialmente en el lado dere-

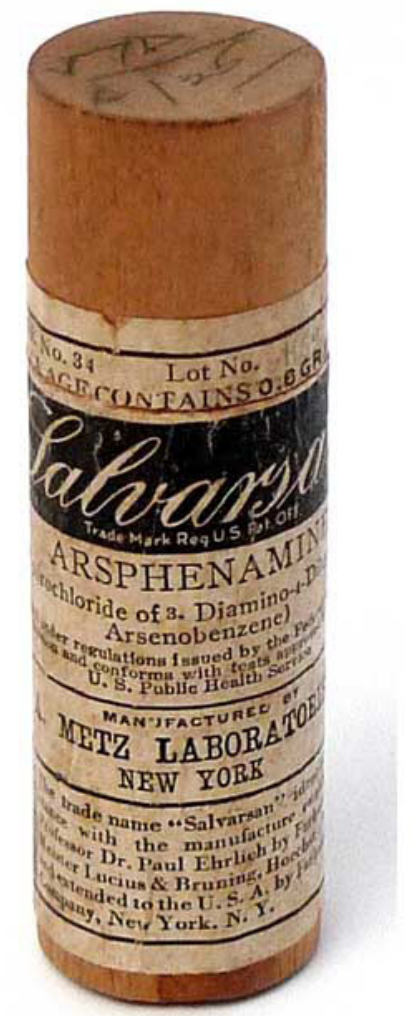

Salvarsán (compuesto 606) elaborado en Nueva York en 1910 por los Laboratorios Metz.

http://www.historiadelamedicina.org/606Expo/ehrlich2.html cho. Desde hace algunos meses presentóse periódicamente un estado febril acompañado de dolor que se propagaba desde el escroto hacia la región inguinal. Tumefacción y enrojecimiento de la piel de las bolsas. Temperatura de $39^{\circ}$ a $40^{\circ}$. Al examen, los linfáticos superficiales turgentes y dilatados, algo endurecidos al tacto, e irregularmente nodulados. La piel algo engrasada y con pequeñas vesículas. Orina. Negativa. Sangre. Microfilaria. Tratamiento. Inyectamos una dosis de 0.60 centígramos de salvarsán en la región glútea. Después de algunos días, en que mejoró el estado del enfermo de la reacción consiguiente a la inyección, desaparecieron los frecuentes accesos de linfangitis retículotroncular. Tratamiento quirúrgico. Practicamos enseguida la resección de las varices linfáticas en ambos lados, precedida de anestesia espinal, y obtuvimos rápida cicatrización de las heridas (Hospital de Caridad).

Caso III (Observaciones del doctor Obregón). Señor M.G.C., de cuarenta y cincos años de edad natural de Cartagena. Sin nada notable en sus antecedentes hereditarios, puesto que nos damos importancia alguna al hecho de que su padre fue un filariasico crónico, desde luego que esta enfermedad no aparece hereditaria; nos hicimos llamar en 1909, para qué asoció al doctor Sergio D. Ibarra, su médico de cabecera, le atendieremos lo que el lenguaje corriente se llamaba en Cartagena una epilepsia de testículo. Efectivamente llegamos al hecho del paciente y encontramos: fiebre de $41^{\circ} \mathrm{C}$ que se ha establecido hace unas 2 horas, después de varios acceso procedidos de escalofríos, cefalea, vómitos, desasosiego y dolor localizado en el escroto, con irradiaciones hacia las ingles. Todo el escroto está rojo e hinchado y cubierto de innumerables vesículas, cuyo tamaño varía desde una cabeza de alfiler hasta el de la yema del dedo. De alguna de estas vesículas, maná una secreción linfática más o menos turbia, clara o lechosa. Los testículos están hinchados y dolorosos a la presión. Los ganglios inguinales están aumentados de volumen.

Prescribimos en asocio de nuestro compañero el rutinario tratamiento de entonces: antitérmicos, medicación antiflogística, an- 
tiséptica local, consistente en compresas empapadas de una solución del bicloruro de mercurio al 1 por 2000, alternadas con otras de resorcina al 1 por 100, quinina per ore, y aventuramos unas tres inyecciones de electrargol. El resultado fue satisfactorio. El enfermo curo como en otras ocasiones, en los innumerables accesos que ha tenido desde hace cuatro años; solo que esta vez la intensidad de los síntomas, la desmesurada hinchazón del escroto con la quilorragia que le acompañaba, justificaron la alarma.

Dos meses después volvimos a ver el enfermo. Estaba bastante bien; las mucosidades patológicas habían desaparecido de la piel del escroto; no existía secreción alguna, si bien había cierto grado de engrosamiento y de lustrosidad de la piel, que es propio de la hipertrofia elefantiásica consecuencial a esta clase de lesiones. Aconsejamos un tratamiento yodurado al interior; una pomada de ictiol y mentol localmente, y perdimos de vista el enfermo por habernos ausentado del país.

Cuatro años después en 1913, fuimos llamados nuevamente para asistir otro ataque análogo al de 1909, y aunque no dudábamos de que el linfoescroto tratado anteriormente era de naturaleza filariosica, no quisimos atenernos a la experiencia clínica, sino que tomamos linfa de una de las vesículas, y allí pudimos descubrir embriones de filaria. Pasada la fiebre y demás síntomas del período agudo de este ataque, quisimos continuar en este caso una tentativa hecha el año anterior en un enfermo del asilo de mendigos, a quién habíamos aplicado una inyección intravenosa de salvarsán, acaso la primera de ese género que se hacía en la ciudad. El resultado en este caso del Asilo fue desastroso, puesto que el enfermo, un caquéctico, murió a los ocho días de inyectado, con síntomas de diarrea, vómitos, edema generalizado y fiebre; pero más instruidos esta vez acerca de la indicaciones y contraindicaciones del Salvarsán, no dudamos en aplicarlo, y al efecto inyectamos una dosis de 60 centigramos, sin otro trastorno que la hiperemia y el prurito del rostro, que era frecuente con los preparados arsénicales que entonces se daban al comercio.
Una semana después de la inyección practicamos un examen nocturno de sangre, y no hayamos filaria. Convencidos de que no es un asunto de fácil encontrar siempre estos parásitos en la sangre, ni prueba concluyente de su existencia el hecho de que no hallarlos en un examen determinado, optamos por tomar un poco de linfa de uno de los viejos quistes del escroto, y el resultado fue positivo. Creímos conveniente por lo tanto repetir la inyección; y al efecto, un mes después hicimos una inyección de igual dosis a la primera. Repetido el examen microscópico al cabo de una semana, no se hallaron filarias a pesar de todo el minucioso cuidado que pusimos en su busca. Con esto coincidió una notable mejoría de todos los síntomas de la enfermedad; y hasta el presente no tenemos noticias de que se hayan presentado nuevos accesos de fiebre ni linfangitis filariásicas.

Nos aseguramos, por su puesto, que no existan filarias en el paciente a que esta observación se refiere; porque así como en ocasiones es difícil hallar las filarias en la sangre, así también pueden aquellas existir al estado latente, sin manifestarse por ningún fenómeno morboso; pero mucho dice en favor de una influencia curativa del salvarsán, la mejoría manifiesta duradera del enfermo.

\section{HEMATOQUILURIA}

Caso I (Observación del doctor Gómez Sánchez). Hemos tenido en estudio un caso de hematoquiluria en un enfermo de sesenta años de edad, natural de Cartagena, que hacía varios años venía sufriendo de accesos hematoquilesos, que se presentaban a intervalos de tiempo más o menos largos (reside actualmente en Barranquilla). Presentóse a nuestro examen con fuerte dolor lumbar, malestar general, estado nauseoso, movimiento febril ligero y dolores vagos en el abdomen. Su orina, algo rojiza al principio, se hizo después perfectamente turbia y lactescente, con coágulos rojizos; de aspecto uniforme recién emitida, se dividía algún tiempo después en tres capas; una superior, cremosa; una media, formada por coágulos opalinorrosaceos, y una tercera, sedimentosa, en la que se encontraban coágulos sanguíneos en considerable 
número. Este aspecto fue modificándose lentamente durante tres meses, hasta quedar perfectamente transparente. Durante las épocas de reposo, la orina tomaba la apariencia normal para enturbiarse pocas horas después de levantada la enferma.

Estos accesos, que hacía varios años venían presentándose, aparecían de ordinario con ocasión de largas exposiciones al sol o excursiones a pie prolongadas. Examen de sangre. Había filarias en número considerable. Examen de orina. Cantidad: 560 gramos. Aspecto: sui generis. Reacción: ligeramente ácida. Urea: 8 gramos. Cloruros: 9 gramos. Fosfatos: 1,20 gramos. Albumina: positiva. Glucosa: negativa. Bilis: negativa. Indicán: pequeña cantidad. Microscopia: hematies en abundancia, leucocitos, células epiteliales, algunos cilindros renales y microfilarias. Para la investigación de estos últimos hemos seguido el método del filtro: recepción de la orina en un embudo provisto de un papel de filtrar, inmediatamente después de emitida. La investigación había sido anteriormente negativa.

La administración de arsenobenzol en este determinado caso no nos pareció prudente durante la época del acceso hematoquilúrico, la presencia de algunos cilindros renales y la avanzada edad del enfermo nos hicieron abstener de su administración. Terminando el acceso y después de haber hecho comprobación de la ausencia de los elementos renales y albúminas, inyectamos intravenosamente 35 centigramos de arsenobenzol. La inyección fue seguida de alguna reacción: malestar general durante varios días, estado nauseoso, pérdida del apetito, dolor lumbar, etc. Este estado fue desapareciendo lentamente, y la enfermedad volvió a su estado normal. Desde que la inyección fue practicada no hemos observado reincidencia alguna, y la enferma ha podido dedicarse libremente a sus ocupaciones habituales.

El segundo examen de sangre fue practicado siguiendo el método de centrifugación, y en dicho examen se encontraron filarias pero en escaso número. Un nuevo examen de orina, realizado quince días después de administrado el medicamento, dio el siguiente resultado: Color: amarillo pálido. Olor: sui géneris. Aspecto: algo turbio. Sedimento: casi nulo. Reacción: poco ácida. Densidad: 1,10. Urea: 10 gramos. Cloruros: 10 gramos. Fosfatos: 2 gramos. Albúmina: negativa. Azúcar: negativa. Indoxilo: negativa. Pigmentos biliares: negativa. Pus: negativa. Sangre: negativa.

Aunque no tenemos derecho para afirmar el alejamiento definitivo de los accesos hematoquilúricos, parécenos que fundadamente podemos pensar que la disminución consiguiente de las microfilarias, la desaparición del dolor lumbar, que era persistente aun después de pasado el acceso, la mejoría del estado general y anémico, son pruebas de cierta importancia de la acción benéfica del arsenobenzol en esta forma clínica de la filariosis.

\section{DERMOLINFANGITIS}

Comprenderemos bajo esta designación anatomopatológica, algunas de las observaciones que vamos a narrar, que corresponden a lo que en Cartagena se designa más comúnmente con el nombre de erisipela.

Sin intención de abordar la oscura cuestión de la patogenia de la lesiones filariósicas, ya que estas líneas estudian una mera cuestión de tratamiento, nos resistimos a aceptar el nombre de erisipela, porque esta palabra despierta el ánimo la idea de estreptococo. Tampoco nos parecen adecuados los términos de fiebre filariásica (filarial fever) de Manson y filariafieberanfall de Scheube), porque ellos designan solamente el síntoma general; ni más aceptables nos parecen las designaciones de elefantiasis filariósicas o de acceso elefantiásico (Forgue y Le Dantec), porque si bien es cierto que la repetición de estos accesos de dermolinfangitis filariósica engendran la elefantiasis, también lo es que no todas las elefantiasis son de origen filariósico.

El término dermolinfangitis filariósica tiene, a nuestro modo de ver, la ventaja de incluir, ya sea el acceso completo con sus fenómenos locales, linfangitis, y generales, fiebre; ya el acceso frustro o atenuado, en que solo se observan fenómenos regionales tales como edema con poco enrojecimiento y ausencia 
de fiebre, como sucede en ciertas formas de elefantiasis de la mano, pie, pierna, etc., (erisipela sorda de Cartagena). Hecha esta pequeña digresión continuamos.

Caso I (observación del doctor Gómez Sánchez). N.N., de veinticinco años de edad, natural de Cartagena y residente en Barranquilla hace algún tiempo. Antecedentes hereditarios. Sin importancia. Antecedentes patológicos. Accesos de angioleucitis reticular en los órganos genitales externos, con fiebre alta $\left(39^{\circ} \mathrm{C}\right)$ y bastante frecuente diagnosticada la filariosis, inyectóse varias veces con hectina y trasladándose a Usiacurí a someterse a tratamiento hidromineral, con el cual obtuvo muy poca mejoría. Practicóse entonces una inyección de 914, que produjo inmediata mejoría. Desaparecieron los frecuentes accesos. Últimamente este enfermo no ha podido ser observado.

Caso II (Observación del doctor Gómez Sánchez). N.N., de treinta y seis años de edad, de raza blanca y constitución fuerte, natural de Cartagena. Antecedentes patológicos. Linfangitis crónica reticular de la mano izquierda, accesos febriles y aspecto erisipelatoso de la piel, que son provocados

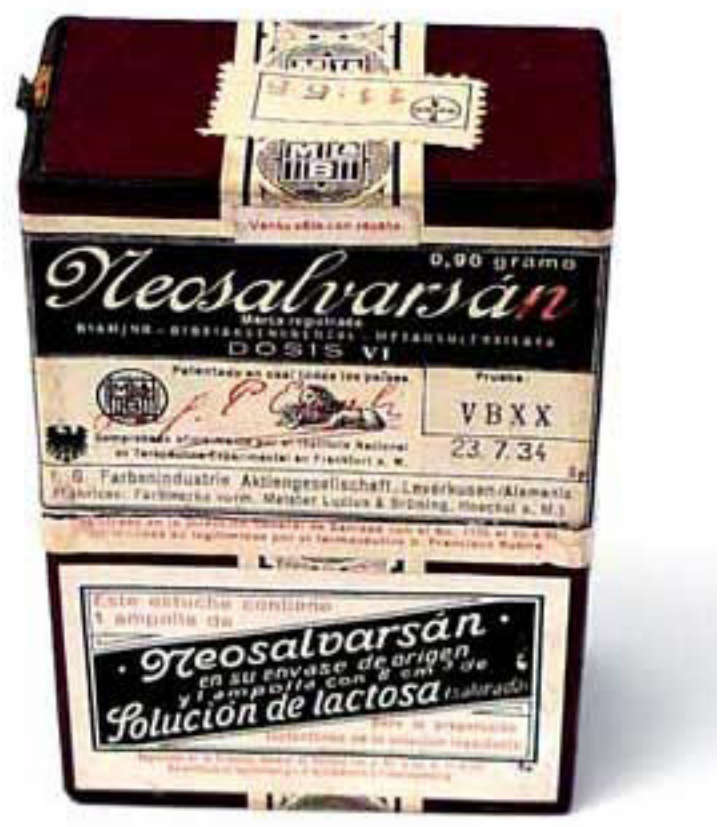

Caja de Neosalvarsán o 914, que apareció en el mercado dos años después que el Salvarsán.

http://www.historiadelamedicina.org/606Expo/ehrlich2.html con el menor ejercicio, exposición al sol, etc. Diagnosticada la filariosis en Cartagena, trasladose a Barranquilla. Practicáronse dos inyecciones intravenosas de salvarsán de 0,45 centigramos cada una, con pocos días de intervalos. Efectos: desaparición de los accesos inflamatorios. Puede dedicarse a cualquier ejercicio por fuerte que sea, y exponerse al sol, sin sentir la molesta sensación de ardor e hiperestesia de la piel, que sentía anteriormente. Desaparición del color violáceo de la piel, que ha adquirido su coloración normal. Disminución del edema crónico. Sangre. Después de cuidadosa centrifugación de la sangre y tratamiento por la solución acética al 2 por 100, el examen ha sido negativo. Orina. Normal. Para modificar la celulitis crónica, inyección de tiodina (tiosinamina y yodo) y fibrolisina. Masajes y vendajes compresivos en los nodos.

El aspecto de la piel se ha modificado, y disminuido considerablemente el edema crónico.

Caso III (Observación del doctor Gómez Sánchez). N.N., de sesenta y tres años de edad, natural de Cartagena y residente en Barranquilla durante quince años.

Antecedentes hereditarios. Sin importancia. Antecedentes patológicos. Sífilis. Linfangitis de repetición en una pierna, en Cartagena, hace algunos años. Posteriormente, como complicación de esta linfangitis, estado inflamatorio de la safena interna. En el periodo de terminación de la flebitis sobrevino una hemiplejia, originada probablemente por alguna emigración trombósica. Hace varios meses los accesos febriles con linfangitis reticular de la pierna sucediéronse regularmente cada mes, sin que se pudiera modificarlos con ningún tratamiento. Previo el examen de orina, y con las precauciones que este especial caso requería (hemiplejia, debilitamiento considerable, edad del enfermo), practicamos una inyección intravenosa de 0,20 centigramos de neosalvarsán. Esta inyección fue precedida, para modificar el estado general, de una de colobiasa de oro. Después de la inyección de 914 no ha reaparecido los accesos, y el estado general ha progresado (aumento de peso). 
Caso IV (Observación del doctor Gómez Sánchez). N.N., de cincuenta y ocho años de edad, natural de Cartagena y vecina de Cereté (rio Sinú), sufre de frecuentes accesos de <erisipela> de la pierna (fiebre filariósica), que se presentan con frecuencia. Orina. Negativa. Tratamiento. Inyección de Flero (emulsión de salvarsán). Obtuvimos notable modificación del estado local y desaparición de los accesos por varios meses. Nota. La enferma se ha perdido de vista hace algún tiempo, y por eso no se puede asegurar la estabilidad de la mejoría.

Caso V. (Observación del doctor Gómez Sánchez). N.N., natural de Cartagena, sufre hace muchos meses de fiebres filariósicas, que se repiten regularmente cada treinta días. Examen de sangre. Microfilaria positiva. Tratamiento. Inyección de 914 intravenosa. Resultado. Desaparición de los accesos de repetición hasta el presente (hacen seis meses). Examen de sangre. Disminución del número de microfilarias. (Examen por el método de centrifugación y solución acética).

Caso VI. (Observación del doctor Obregón). Señorita N.N., treinta y dos años de edad; hace quince años vive en Cartagena. Contrajo la<erisipela> hace dos años, la cual se ha manifestado periódicamente bajo la forma de placas erisipeloides que ocupan siempre el dorso del pie derecho. No recuerda de circunstancia ninguna determinante para la producción de su enfermedad, aunque le parece que esta tiene cierta relación con el flujo catamenial, porque casi siempre le atacan los accesos cuando está para enfermar.

El 23 de enero de 1914 me hace llamar porque el acceso ha cambiado de sitio y esta vez aparece en el pie izquierdo, prolongándose la inflamación hasta la parte media de la cara externa de la pierna correspondiente. Temperatura $40.5^{\circ} \mathrm{C}$; calofríos frecuentes han precedido a esta temperatura que no ha presentado remisiones apreciables. Bazo e hígado, normales. Orina marcada indicanuria. Tratamiento sintomático: el 26 hacemos un examen nocturno de sangre, que fue negativo. El 28, nuevo examen centrifugando en solución acética al 2 por 100 , e igual negativo el resultado.
No conformes con esto, porque partíamos de la idea de que en un caso tan típico necesariamente debía existir microfilarias, apelamos el 5 de febrero al procedimiento de Nattan-Larrier y Bergeren, procedimiento que a grandes rasgos describiremos enseguida, por ser poco conocido y porque de cuantos hemos empleado para la busca de las microfilarias, es el que reputamos más seguro y menos enojoso para el paciente, pues no se necesita que la sangre sea tomada de noche.

Con una jeringuilla aséptica se toman 10 centímetros cúbicos en cualquiera de las venas del pliegue del dedo; se coloca esta sangre por partes iguales en dos frascos bien limpios que contengan 100 gramos de agua esterilizada y destilada; se disuelve bien la sangre para el efecto de hemolizar; el contenido de los frascos se reparte en tubos de centrifugar; se hace rotar la maquina durante un cuarto de hora, y de los residuos depositados en el fondo de los tubos se hacen varias preparaciones que se fijan por vapores de ácido ósmico o se colorean por cualquiera otro medio de los conocidos. Innumerables veces nos hemos valido de este procedimiento, y nunca lo hemos visto fallar, aun en caso en que los otros han fallado. Esto nos revela de decir que a favor de este procedimiento pudimos comprobar la existencia de la microfilaria en el caso de que tratamos. Hecha esta comprobación, procedimos a inyectar por vía intravenosa 0,60 centigramos de salvarsán. Quince días después, nuevo examen de sangre que fue negativo. Por parecernos dudoso el examen inyectamos 0,40 centigramos para mayor seguridad. La enferma continúo bien.

En mayo del presente año volvimos a verla. El examen de la sangre, con gran sorpresa nuestra, fue positivo, que de seis placas, en cuatro encontramos microfilarias; pero la enferma no ha tenido en todo el tiempo transcurrido desde la última inyección de salvarsán un solo acceso de fiebre filárica.

Interpretamos este caso admitiendo que el efecto de la inyección no es permanente; que la enferma se ha reinfectado por continuar viviendo en las mismas condiciones de receptividad morbosa; y que si no ha tenido 
nuevos ataques es por estar la enfermedad en estado latente. Ninguna importancia damos a la coincidencia de los accesos con el periodo menstrual, porque es sabido que la filariosis no tiene ciclo conocido; la mayor parte de las veces la enfermedad se desarrolla, aunque no constantemente, bajo la forma de accesos frecuentes o irregulares, a intervalos de semanas, meses y años. (Scheube. Die krankheiten der Warner Lander, página 438).

Caso VI (Observación del doctor Obregón). Señora V.V., veintiséis años. Ha tenido varios partos felices. Su madre ha sufrido de dermolinfangitis con repetición, lo que ha dado por consecuencia cierto grado de elefantiasis de ambas piernas. Antecedentes patológicos. Dermolinfangitis de repetición, lo mismo que la madre, con quien ha vivido todo el curso de su vida, en las mismas condiciones higiénicas. Las manifestaciones filariósicas le vinieron hace un año, mucho tiempo después de casada. La convivencia en que ha crecido nos dispersa de dar valor herencial alguno al estado patológico actual, desde luego que esta enfermedad no tiene por qué ser hereditaria.

Asistimos a la enferma en momentos en que acaba de pasar uno de sus habituales ataques. La pierna izquierda, en el punto afectado, aún está rojiza, lustrosa y edematosa: es todo cuanto dice el examen físico. El primer examen de sangre fue negativo desde el punto de vista parasitológico; no obstante encontramos un ligero grado de anemia secundaria caracterizado por oligocitemia $(3.800,000)$, oligoromemia (80 por 100), algunos polikilicitos; la siguiente formula leucocitaria: linfocitos: 30 por 100 . Grandes mononucleares: 25 por 100. Polinucleares: 21.5 por 100 . Eosinófilos: 13.5 por 100 .

La eosinofilia nos indica que debe existir filaria; y el segundo examen de sangre, practicado por el método de Nattan-Larrier, se encargada de ratificarlo. Con esta certeza inyectamos una dosis de 0,70 centigramos de neosalvarsán. Resultado: durante de tres meses ausencia de ataques erisipeloides que antes sobrevenían casi regularmente cada mes.
En presencia de este fracaso relativo, resolvimos hacer una nueva inyección de una ampolla completa de neosalvarsán $(0,90)$, lo cual verificamos a los seis meses de la primera inyección, debido a las vacilaciones de la enferma; y anotamos estas circunstancias como prueba de lo difícil que es la observación científica entre nosotros, siendo quizás el menor de los inconvenientes la renuencia de los pacientes.

El resultado de esta última inyección es el que hace siete meses la enferma no ha tenido un solo ataque; la piel de la pierna, que comenzaba a paquidermizarse, ha recobrado su blandura natural; y la enferma, en lo general, goza de buena salud.

Caso VII (Observación del doctor Obregón). V.A., señora de veinticuatro años de edad, hermana de la paciente de la anterior observación. Los mismos antecedentes hereditarios y unas mismas condiciones higiénicas. Hacen dos años tuvo un ataque de fiebre filárica con angiólencitis correspondiente. Hasta el presente (1917) no ha vuelto a tener nada semejante; pero de cuando en cuando suele presentársele en el pliegue del codo derecho, y extendida del brazo al antebrazo, una placa gruesa, ligeramente dolorosa, pero sin enrojecimiento ni reacción inflamatoria notable, la que desaparece en dos o tres días y deja todo en su primitivo estado. En resumen: una linfangectasia cutánea producida por alguna obstrucción de los linfáticos profundos (<erisipela sorda $>$ ).

Comprobada la microfilaria por el examen de laboratorio, y al mismo tiempo una polinucleosis con eosinofilia, inyectamos por la técnica de Ravaut, que es la que seguimos desde hace tres años en todas las inyecciones de salvarsán, 0,50 centigramos de novarsenobenzol de Billen. Dos meses después hemos practicado nuevo examen hematológico, y no hemos hallado microfilarias, sino más bien que la sangre ha recuperado sus condiciones normales, lo cual anotamos como un triunfo más del arsenobenzol.

Caso VIII (Observación del doctor Obregón). Señorita J.M., cuarenta y cuatro años de 
edad. A los diez y nueve años tuvo un acceso de linfodermitis del dorso del pie izquierdo, que repitió en dos ocasiones a un mes más o menos de intervalo sin dejar huellas. Cuatro años de calma, al cabo de los cuales tuvo otro acceso. Un año después otro. Transcurrieron diez y siete años en completo estado de salud, y al cabo de ellos se presentó otro acceso. Se ausentó entonces de la localidad, y de regreso un año después, ha tenido otro acceso (mayo de 1917); pero este último se ha verificado, con idénticos caracteres a los anteriores, en el pie derecho. Total: siete accesos en veinticinco años, a grandes intervalos, entre los cuales se cuenta un periodo de diez y siete años.

Esta paciente ha vivido siempre en muy buenas condiciones higiénicas y ha viajado bastante, lo cual explicaría la poca frecuencia de los accesos. Comprobada la filariosis, inyectamos 0,60 centigramos de novarsenobenzol, sin que hasta el presente (noviembre), o sean seis meses después, se haya presentado un nuevo acceso.

Se comprende, desde luego, que, dada la modalidad patológica de esta paciente, ninguna conclusión podemos sacar acerca de la eficacia del tratamiento por el salvarsán, tanto más cuanto la enferma continua aun en observación; mas hemos apuntado el caso en el presente trabajo, por lo interesante que parece la irregularidad de los ataques.

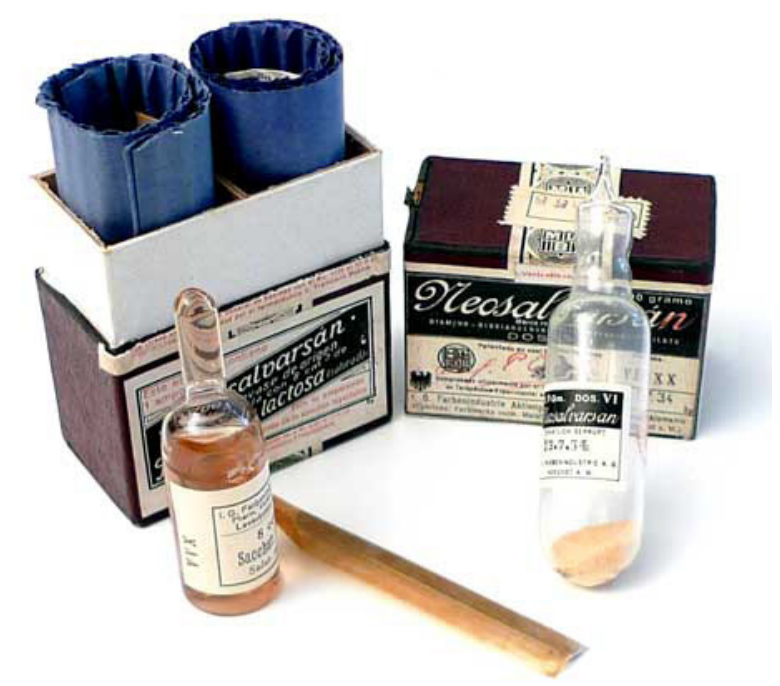

Neosalvarsán inyectable (compuesto 914).

http://www.historiadelamedicina.org/606Expo/ehrlich2.html
Todavía disponemos de una veintena de observaciones más pertenecientes a este grupo de dermalinfangitis, tratadas por arsenobenzol; pero en gracia de la brevedad queremos dejar constancia solamente del resultado, el cual no siempre ha sido favorable con una sola inyección, especialmente si el enfermo no ha variado de condiciones de vida.

\section{HIDROCELE QUILOSA}

Caso I (Observación del doctor Obregón). L.C.R., de treinta y ocho años de edad, natural del departamento de Boyacá, color moreno, soltero. Hace dos años, poco más o menos, que llegó a la costa con el empleo de agente de policía marítimo. Entró al hospital en esta ciudad el día 11 de septiembre de 1917, y ocupó la cama número $6^{\mathrm{a}}$. Antecedentes hereditarios. No los conoce, pues ignora que enfermedades hayan tenido sus padres y familiares. Antecedentes personales. Es persona robusta y siempre ha gozado de buena salud. En el interior de la Republica sufrió hace mucho tiempo dos ataques de erisipela estreptocóccica (al decir de los médicos que lo atendieron), por los cuales no hizo cama, según fueron de benignos.

Hace poco más o menos un año sufrió un pequeño traumatismo en el testículo izquierdo, accidente de poca consideración al cual no le dio importancia; más poco tiempo después notó que el testículo comenzaba a crecer a la vez que se le hacía doloroso. En esta situación estaba cuando lo mandaron para Zapzurro, donde adquirió las fiebres que lo han traído al hospital. Estado actual. Fiebres francamente intermitentes y de naturaleza palúdica, según lo ha comprobado el examen. Se ha instituido el tratamiento conveniente. Se ha encontrado además: tumor globuloso, fluctuante, de tamaño medio, que ocupa la totalidad de la celda correspondiente al testículo traumatizado. Poca y casi ninguna transparencia en el tumor; a pesar de esto se hizo el diagnostico de hidrocele, basándose para ello en el examen de sangre, que fue positivo en hemamebas palúdicas y en filarias.

Orina. Confiesa el enfermo haber emitido orinas lechosas por varias ocasiones durante varios días; pero examinadas actualmente; se ha encontrado que son normales. El aspecto 
de las orinas y ciertos dolores lumbares que sentía, le hicieron suponer que sufría de los riñones; y al efecto se hizo algunos remedios por su propia cuenta.

Se trató de operarlo; mas como le daban fiebres, se resolvió aguardar a que estas desaparecieran por completo. Durante este tiempo se le inyecto otoxil, mejoro y salió del hospital sin operarse. El día 5 de octubre volvió al hospital, quejándose entonces de la molestia que le causaba la tensión dolorosa del testículo. El 7 del mismo mes se le practicó una inyección de 0,60 centigramos de novarsenobenzol Billón. En espera de resultado que ella tuviera, se resolvió al fin de hacerle la inversión de la vaginal, operación que fue practicada por el doctor Nicolás $M$. Paz, en asocio del autor de esta observación. Al hacer la incisión de la serosa, se encontró un líquido lechoso que, recogido y examinado, dio muestra de filaria. (Placa presentada). Siete días después de operado, el 29 de octubre, se le hizo una inyección de 0,45 centigramos de novarsenobenzol alemán.

Consecuencias de la operación, normales; cicatrización por primera intención. 13 de noviembre. Examen de sangre, negativo desde el punto de vista de la filaria. Sangre tomada por picadura nocturna. 20 de noviembre. Examen de sangre, negativo sangre tomada en la vena.

Por las historias clínicas que dejamos sucintamente narradas, es innegable que el salvarsán ejerce alguna influencia favorable en la mayor parte de las manifestaciones filariósicas; pero antes de llegar a más determinadas conclusiones se nos ocurre preguntar. ¿Obra el medicamento destruyendo el parasito adulto o los embriones? Esta cuestión, a la verdad, no es fácil de resolver por el momento, porque excepto las investigaciones de Mouneyrat y de Tanen acerca de la acción de algunos derivados arsenosulfurados hechas en ciertas trepanesomiasis espirilosis experimentales, no sabemos de estudios comparativos que se hayan hecho en la cuestión que tratamos. Para Mouneyrat y Tanen, la mayor parte de esos compuestos arsenosulfurados tienen una acción manifiesta tanto en los espirilos como en los tripanosomas,

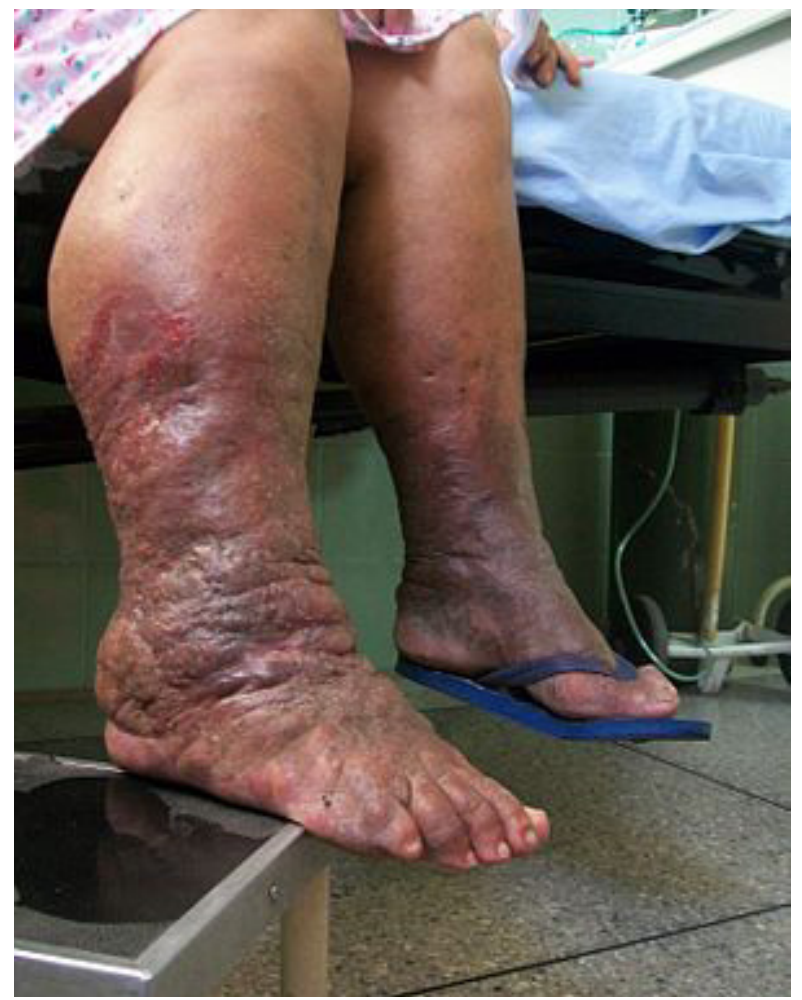

Elefantiasis. Mujer de 69 años de edad.

https://en.wikipedia.org/wiki/Lymphedema

lo que ha hallado comprobación exacta en el tratamiento de la fiebre recurrente por el salvarsán; sin embargo es de suponerse que el salvarsán tenga una acción diferente no solo entre parásitos de especie diversa como de filarias a espirilos, sino entre parásitos de la misma especie; puesto que la acción esterilizante del medicamento en la sangre ha de depender en suma del poder de resistencia que tiene que ser variable como las condiciones biológicas de cada organismo.

Es la sífilis la enfermedad en que el salvarsán ejerza su máximo de acción y alcanza su mayor eficacia; sin embargo, desde los ensayos de Iversen para acá, Bitter y Dreyer, Ardin-Denteil y Raynaud, han demostrado que arsenobenzol es el medicamento especifico de la fiebre recurrente, sin igual en la farmacopea; que en pocas horas ejerce una acción esterilizante tan notable del organismo, que no se encuentra en la sangre un solo parasito; que esta acción es tan rápida que casi puede considerarse como abortiva, y por lo mismo, casi nunca hay que repetir la dosis del medicamento. 
Bien sabemos que de la sífilis no se puede decir lo mismo; y eso que ambos casos se trata de espirilos.

Nosotros hemos ensayado el salvarsán en la filariosis, con el mismo intento que otros lo han ensayado en el pénfigo, la lepra, el kala-azar, la malaria, frambuesia, bilharzia, etc.; y hemos obtenidos resultados bastante satisfactorios; pero en ningún caso podríamos aseverar que esos resultados sean tan notables como los alcanzados en la sífilis o en el tifus recurrente, ya que ni todos los casos ceden al salvarsán ni siempre basta una sola inyección.

Posible es que esto último se deba a que el enfermo una vez curado sigue viviendo en el mismo medio y sometido a las mismas influencias patológicas; pero en todo caso nuestro deber es dejar constancia del hecho. Pero volvamos a nuestra pregunta: ¿sobre cuál de los organismos ejerce su acción el arsenobenzol, la filaria o la microfilaria? Según Dopter, las microfilarias no tienen acción patógena. De la misma opinión es Manson, quien dijo muchísimos antes de Dopter: "the healty, fulle-fermed embryofilariae-that is tos ay, the filariae which, by means ot the microscope, we see in the blood-have, se far as we cantrll, no pathogenic properties whatever". Solo la forma adulta y sus productos no maduros son peligrosos. Siendo esto así, es de suponerse que el salvarsán ejercerá su acción sobre la filaria adulta y no sobre los embriones, que serán arsenorresistentes. Si esta hipótesis tiene algún fundamento, vendría ella a explicar el porqué de la mejoría observada en algunos casos, después de la inyección del salvarsán, aun cuando todavía existen microfilarias en la sangre.

Es creencia general en Cartagena que cuando la placa erisipeloide se supura, lo que desde luego puede deberse a varios agentes patógenos, la curación es espontánea y definitiva. En este caso podemos suponer que la muerte accidental del parasito adulto y la disgregación de su cadáver provocan el absceso local de fijación que se encarga de eliminar, juntos con los elementos disgregados, los demás productos patógenos formados.

Es bien posible pues que la muerte del parasito adulto y la consecuente eliminación de los productos tóxicos que el elabora, sean el fundamento de la acción curativa del salvarsán. En tal caso de los embriones desaparecen por fagocitosis o por esterilización que el medicamento efectúa en el organismo; y solo persistirían las lecciones de carácter permanente que la enfermedad ocasionara en los vasos linfáticos y el tejido conjuntivo. A esta reparación contributaria en mucho la benéfica influencia que el arsenobenzol ejerce en la constitución de la sangre, demostrado como esta por Weill Guenot que dicho medicamento es un poderoso renovador sanguíneo porque aumenta el número de hematias y la resistencias de los mismos a la hemolisis, y aumenta también la cantidad de la hemoglobina.

Expuesto lo anterior, nos permitimos sentar las siguientes conclusiones $d$ 'attente, mientras estudios posteriores les den o no sanción definitiva:

Primera. El arsenobenzol es el mejor tratamiento que al presente tiene la ciencia médica para la modificación de la terrible endemia de Cartagena.

Segunda. Es en las angioleucitis filiariosicas «de repetición» donde el salvarsán produce sus mejores y más brillantes resultados. Esta convicción llegó al ánimo de uno de nosotros (doctor Gómez Sánchez), después de haber inyectado en julio de 1912 un joven filariósico afectado de angioleucitis escrotal «a repetición» manifestación de la filaria que después de continuos y repetidos accesos lleva faltamente a la elefantiasis progresiva de la región asiento de los brotes inflamatorios.

Tercera. El arsenobenzol - y nótese que decimos adrede salvarsán o arsenobenzol, porque hemos usados indistintamente los dos preparados sin hallar las notables diferencias que tanto han desvelados a otros observadores-ejerce benéfica influencia en la hematoquiluria y el linfoescroto, asi como en otros estados filariósicos. 
Cuarta. La influencia del medicamento en cuestión es nula en ciertas lesiones definitivas: elenfantiasis, adenitis inguinal filariósica (varirose leistendrusen de Scheube), varices linfáticas, etc., que requieren, ya la intervención quirúrgica, ya la de agentes fribilizantes (tiodina, tiosinamina, etc.).

Quinta. A semejanza de lo que pasa en el tratamiento de las espirilosis, salvo la fiebre recurrente, la administración del medicamento debe ser precoz e intensiva, pues la mayor parte de las veces no basta una fuerte dosis administrada por una sola vez.
Sexta. La benéfica acción del salvarsán en los estados anémicos por la influencia química del arsénico sobre sobre la médula ósea (trabajos de Weill y Luis Guenot), es una razón más para usarlo en la filariosis.

Séptima. Los casos negativos que puedan encontrarse en la práctica no son objeciones serias para el uso frecuente del medicamento, porque igualmente los hallamos en las espirilosis y tripanosomiasis, lo cual ha explicado Ehrlich, por la formación en razas de parásitos arsenorresistentes.

Cartagena, noviembre de 1917.
Es el órgano de información cientifica de la Facultad de Medicina de la Universidad de Cartagena. Colombia.

Publique su trabajo en esta revista enviando su manuscrito a: revistacienciasbiomedicas@unicartagena.edu.co http://revistas.unicartagena.edu.co/index.php/cienciasbiomedicas www.revistacienciasbiomedicas.com.co

Revista ciencias Biomédicas es una publicación independiente, imparcial, abierta, revisada por pares, de elevada visibilidad internacional, con circulación online e impresa. Publica articulos en todas las modalidades universalmente ciencias biomédicas, incluyendo ámbitos clínicos, epidemioógicos o estudios básicos.

El sistema de gestión de manuscritos es rápido y justo.

Revista Ciencias Biomédicas está incluida en varias bases de datos latinoamericanas e internacionales.

Antes de enviar su manuscrito, revise las recomendaciones para los autores, presentes en: http://revistas.unicartagena.edu.co/index.php/cienciasbiomedicas www.revistacienciasbiomedicas.com.co 